\title{
On the Numerical Solution of Some Eikonal Equations: An Elliptic Solver Approach*
}

\author{
Alexandre CABOUSSAT ${ }^{1}$ Roland GLOWINSKI ${ }^{2}$ Tsorng-Whay PAN ${ }^{2}$ \\ (In Honor of the Scientific Contributions of Professor Luc Tartar)
}

\begin{abstract}
The steady Eikonal equation is a prototypical first-order fully nonlinear equation. A numerical method based on elliptic solvers is presented here to solve two different kinds of steady Eikonal equations and compute solutions, which are maximal and minimal in the variational sense. The approach in this paper relies on a variational argument involving penalty, a biharmonic regularization, and an operator-splitting-based time-discretization scheme for the solution of an associated initial-value problem. This approach allows the decoupling of the nonlinearities and differential operators.

Numerical experiments are performed to validate this approach and investigate its convergence properties from a numerical viewpoint.
\end{abstract}

Keywords Eikonal equations, Maximal solutions, Regularization methods, Operator splitting, Finite element methods

2000 MR Subject Classification 35F30, 49M20, 65K10, 65M60, 65N30

\section{Introduction}

Various mathematical models in science and engineering lead to the Eikonal equation; this is the case particularly in optics, wave propagation, material science, differential geometry (geodesics) (see [9]), geophysics (see [11]), and image processing (see [10, 13]). The analysis of such nonlinear models can be found in [4] (see also the references therein). Actually, the Eikonal equation is often related with the Hamilton-Jacobi equation for wave propagation (see [14]).

The goal of this article is to discuss the numerical solution of the Dirichlet problem for some steady Eikonal equations in two dimensions of space, using elliptic solvers-based methodologies and low order, finite element approximations. The main problem we want to address is a steady scalar Eikonal equation: Find $u: \Omega \subset \mathbb{R}^{2} \rightarrow \mathbb{R}$ verifying

$$
\begin{cases}|\nabla u|=1 & \text { in } \Omega, \\ u=g & \text { on } \partial \Omega .\end{cases}
$$

\footnotetext{
Manuscript received December 1, 2013.

${ }^{1}$ Haute Ecole de Gestion de Genève, University of Applied Sciences, Western Switzerland (HES-SO),

Route de Drize 7, 1227 Carouge, Switzerland. E-mail: alexandre.caboussat@hesge.ch

${ }^{2}$ Department of Mathematics, University of Houston, 4800 Calhoun Rd, Houston Texas 77204-3008,

USA. E-mail: roland@math.uh.edu pan@math.uh.edu

*This work was supported by the National Science Foundation (No. DMS-0913982).
} 
The approach via elliptic solvers we advocate goes against the common wisdom for this type of problems, which is to address their numerical approximation via viscosity solutions and hyperbolic equation methods. However, one can see three main reasons to justify the use of such an approach:

(i) The solutions of (1.1) (and later (1.3)) are Lipschitz continuous but do not have, in general, a $W^{2, p}$-regularity. This strongly suggests the use of globally continuous piecewise affine finite element approximations.

(ii) It was shown by Caffarelli and Crandall [1] that, away from the singularities of $\nabla u$, the solutions of the Eikonal equation $|\nabla u|=1$ are piecewise affine or conical.

(iii) The elliptic solver approach appears to be well-suited to the solution of Eikonal systems such as the following natural extension of (1.1): Find $\mathbf{u}: \mathbb{R}^{2} \rightarrow \mathbb{R}^{2}$ satisfying

$$
\begin{cases}\nabla \mathbf{u}(\mathbf{x}) \in \mathcal{O}(2) & \text { a.e. in } \Omega \\ u=g & \text { on } \partial \Omega\end{cases}
$$

where $\mathcal{O}(2)$ denotes the set of $2 \times 2$ orthogonal matrices. Systems such as (1.2) were considered in origami theory (see for instance [5-6]). There exist very efficient elliptic solvers making use of globally continuous piecewise affine finite element approximations. Using such solvers to solve (1.1) would provide thus an alternative methodology to the marching techniques advocated by Osher and Sethian [14]. In parallel, we can apply the methodology, with some modifications described hereafter, to the following Eikonal system: Find $u: \Omega \subset \mathbb{R}^{2} \rightarrow \mathbb{R}$ verifying

$$
\begin{cases}\left|\frac{\partial u}{\partial x_{1}}\right|=\left|\frac{\partial u}{\partial x_{2}}\right|=1 & \text { in } \Omega, \\ u=g & \text { on } \partial \Omega,\end{cases}
$$

whose solutions may show some multi-scale properties related to a fractal behavior (see [2-3]).

This article is organized as follows: In Sections $2-3$, we formally describe the Eikonal equations which we are interested in and the corresponding problems from Calculus of Variations for which we address to obtain a maximal solution. Section 4 contains the technical details about our penalization/regularization method, as well as the definition of the corresponding initial-value problem. Section 5 describes the splitting algorithm and the solution methods used at each step. Finally, the results of numerical experiments are presented in Section 6 .

\section{Two Eikonal Problems}

The goal of this work is to discuss the numerical solution of the Dirichlet problem for some steady Eikonal equations, using an elliptic solver based methodology. Letting $\Omega$ be a bounded domain of $\mathbb{R}^{2}$, we suppose that the boundary $\Gamma:=\partial \Omega$ is Lipschitz continuous. The steady scalar Eikonal equation which we want to solve reads as follows: Find $u: \Omega \rightarrow \mathbb{R}$ verifying

$$
\begin{cases}|\nabla u|=1 & \text { in } \Omega, \\ u=g & \text { on } \Gamma .\end{cases}
$$

The function $g$ is assumed to be sufficiently smooth. Up to the addition of a constant, we can always suppose that $g \geq 0$. Since problem (2.1) may have infinitely many solutions, it makes sense to enforce uniqueness by imposing additional conditions. In this article, we will impose on 
the solution to be maximal in the sense of $L^{1}(\Omega)$ (actually, we can also impose on the solution to be minimal).

On the other hand, we consider another steady Eikonal equation (see [2]): Find $u: \Omega \rightarrow \mathbb{R}$ verifying

$$
\begin{cases}\left|\frac{\partial u}{\partial x_{1}}\right|=\left|\frac{\partial u}{\partial x_{2}}\right|=1 & \text { in } \Omega, \\ u=g & \text { on } \Gamma .\end{cases}
$$

Again for this problem, the notion of maximal/minimal solutions holds. However, simple examples described in Section 6 stress clearly the same fundamental differences between problems (2.1) and (2.2); indeed, problem (2.2) may have the maximal or minimal solutions with a fractal behavior for $g=0$, unlike problem (2.1).

\section{Problems of Calculus of Variations}

Since (2.1) may have infinitely many solutions, a particular attention will be given to the maximal (resp. minimal) solutions with respect to some criterion, to force uniqueness. We formulate this problem as a constrained minimization problem from Calculus of Variations, and use the very rich computational methodology associated with this type of problems to solve it. In order to obtain the maximal solution of (2.1), we thus require $u$ to maximize the linear functional

$$
v \rightarrow \int_{\Omega} v \mathrm{~d} \mathbf{x}
$$

over

$$
E_{g}=\left\{v \in H^{1}(\Omega),|\nabla v|=1 \text { in } \Omega, v=g \text { on } \Gamma\right\},
$$

which is actually equivalent to maximizing the $L^{1}$-norm over $E_{g}$. We can easily prove that the above function is also the upper hull of the functions in $E_{g}$, that is, the (necessarily unique, see, e.g., [12]) function $u$ of $E_{g}$ verifying

$$
u(\mathbf{x}) \geq v(\mathbf{x}), \quad \forall v \in E_{g}, \quad \forall \mathbf{x} \in \Omega .
$$

Remark 3.1 In order to find the smallest solution of (2.1) in the sense of (3.3), that is

$$
u(\mathbf{x}) \leq v(\mathbf{x}), \quad \forall v \in E_{g}, \forall \mathbf{x} \in \Omega,
$$

we would minimize over $E_{g}$ the functional $v \rightarrow \int_{\Omega} v \mathrm{~d} \mathbf{x}$.

Remark 3.2 If $g=0$, the maximizer of (3.1) is nothing but $\mathbf{x} \rightarrow \delta(\mathbf{x}, \Gamma)$, the distance function to the boundary $\partial \Omega$. For example, in one dimension of space, if $\Omega=(0,1)$, then $u(x)=\min (x, 1-x)$; in two dimensions of space, if $\Omega=(0,1) \times(0,1)$, then

$$
u(\mathbf{x})=u\left(x_{1}, x_{2}\right)=\min \left(x_{1}, x_{2}, 1-x_{1}, 1-x_{2}\right)
$$

for all $\mathbf{x}=\left\{x_{1}, x_{2}\right\} \in \Omega$. If $\Omega$ is the disk of radius $R$ centered at 0 , then $u(\mathbf{x})=u\left(x_{1}, x_{2}\right)=$ $R-\sqrt{x_{1}^{2}+x_{2}^{2}}$, a typical conical function. 
In the remainder of this article, we describe a numerical method for the computation of approximations of the maximal and minimal solutions in the general case. In order to do so, let $C>0$ be a given positive constant and note there is an equivalence between

$$
u=\arg \max _{v \in E_{g}} \int_{\Omega} v \mathrm{~d} \mathbf{x}
$$

and

$$
u=\arg \min _{v \in E_{g}} J(v)
$$

with

$$
J(v)=\left[\frac{1}{2} \int_{\Omega}|\nabla v|^{2} \mathrm{~d} \mathbf{x}-C \int_{\Omega} v \mathrm{~d} \mathbf{x}\right] .
$$

The main difficulty with (3.6) is the nonlinear constraint $|\nabla v|=1$ (which is equivalent to $\left.|\nabla v|^{2}=1\right)$.

Remark 3.3 In order to capture the minimal solution instead of the maximal solution, it suffices to change $C$ in $-C$ into (3.6), $C$ still being a strictly positive constant.

When addressing (2.2), the approach remains similar. The variational problem can be written as

$$
\bar{u}=\arg \min _{v \in E_{g}^{+}} J(v),
$$

where

$$
E_{g}^{+}=\left\{v \in H^{1}(\Omega),\left|\frac{\partial v}{\partial x_{i}}\right|=1, i=1,2, v \geq 0, \text { in } \Omega, v=g \text { on } \Gamma\right\} .
$$

Note that, for stability reasons, we introduce the additional constraint $v \geq 0$ for this second problem to avoid solutions with oscillations taking negative values close to the boundary.

\section{Penalty/Regularization Approach}

Motivated by Ginzburg-Landau models, we are going to treat $|\nabla v|=1$ by an exterior penalty method preserving the differentiability of the cost functional. Moreover, in order to

control mesh-related oscillations and improve robustness, we are going to bound $\left\|\nabla^{2} u\right\|_{L^{2}(\Omega)}$ (without this additional constraint our method may not work for some test cases, especially for $(2.2))$. Letting $\varepsilon_{1}, \varepsilon_{2}>0$ be small parameters and denote $\left\{\varepsilon_{1}, \varepsilon_{2}\right\}$ by $\varepsilon$, we approximate thus (3.6) by the problem

$$
\min _{v \in V_{g}}\left[J(v)+\frac{\varepsilon_{1}}{2} \int_{\Omega}\left|\nabla^{2} v\right|^{2} \mathrm{~d} \mathbf{x}+\frac{1}{4 \varepsilon_{2}} \int_{\Omega}\left(|\nabla v|^{2}-1\right)^{2} \mathrm{~d} \mathbf{x}\right],
$$

where $V_{g}=\left\{v \in H^{2}(\Omega), v=g\right.$ on $\left.\Gamma\right\}$, and we denote by $u_{\varepsilon} \in V_{g}$ its solution. Using compactness arguments, we can show that (4.1) has a solution, the main difficulty being to compute it. In order to make the computations simpler, we are going to transfer the nonlinearity burden to $\mathbf{p}_{\varepsilon}:=\nabla u_{\varepsilon}$ via the following equivalent mixed formulation of (4.1):

$$
\mathbf{p}_{\varepsilon}=\arg \min _{\mathbf{q} \in \mathbf{Q}}\left[\frac{1}{2} \int_{\Omega}|\mathbf{q}|^{2} \mathrm{~d} \mathbf{x}-C \int_{\Omega} \nabla \varphi_{1} \cdot \mathbf{q} \mathrm{d} \mathbf{x}+\frac{1}{4 \varepsilon_{2}} \int_{\Omega}\left(|\mathbf{q}|^{2}-1\right)^{2} \mathrm{~d} \mathbf{x}+I_{\nabla}(\mathbf{q})\right],
$$


where

(i) $\mathbf{Q}=\left\{\mathbf{q} \in L^{2}(\Omega)^{2}\right\}$;

(ii) the function $\varphi_{1}$ is the unique solution in $H^{1}(\Omega)$ of the Poisson-Dirichlet problem:

$$
\begin{cases}-\nabla^{2} \varphi_{1}=1 & \text { in } \Omega \\ \varphi_{1}=0 & \text { on } \Gamma\end{cases}
$$

(iii) the functional $I_{\nabla}(\cdot)$ is defined by

$$
I_{\nabla}(\mathbf{q})= \begin{cases}\frac{\varepsilon_{1}}{2} \int_{\Omega}|\nabla \cdot \mathbf{q}| \mathrm{d} \mathbf{x}, & \text { if } \mathbf{q} \in \nabla V_{g}, \\ +\infty, & \text { if } \mathbf{q} \in \mathbf{Q} \backslash \nabla V_{g} .\end{cases}
$$

The functional $I_{\nabla}(\cdot)$ is convex, proper and lower semi-continuous.

The Euler-Lagrange equation associated with the minimization problem (4.2) reads as follows, when written in the variational form. For simplicity, the subscript $\varepsilon$ will be dropped from now on. Find $\mathbf{p} \in \mathbf{Q}$ satisfying

$$
\int_{\Omega} \mathbf{p} \cdot \mathbf{q} \mathrm{d} \mathbf{x}+\frac{1}{\varepsilon_{2}} \int_{\Omega}\left(|\mathbf{p}|^{2}-1\right) \mathbf{p} \cdot \mathbf{q} \mathrm{d} \mathbf{x}+\left\langle\partial I_{\nabla}(\mathbf{p}), \mathbf{q}\right\rangle=C \int_{\Omega} \nabla \varphi_{1} \cdot \mathbf{q} \mathrm{d} \mathbf{x}
$$

for all $\mathbf{q} \in \mathbf{Q}$.

The next step is to associate with (4.4) an initial-value problem (flow in the dynamical system terminology), and time-discretize this initial-value problem by an operator-splitting scheme. The initial-value problem reads as follows: Find $\mathbf{p}(t) \in \mathbf{Q}$ for a.e. $t \in(0,+\infty)$ satisfying

$$
\left\{\begin{array}{l}
\int_{\Omega} \frac{\partial \mathbf{p}}{\partial t} \cdot \mathbf{q} \mathbf{d} \mathbf{x}+\int_{\Omega} \mathbf{p} \cdot \mathbf{q} \mathrm{d} \mathbf{x}+\frac{1}{\varepsilon_{2}} \int_{\Omega}\left(|\mathbf{p}|^{2}-1\right) \mathbf{p} \cdot \mathbf{q} \mathrm{d} \mathbf{x} \\
\quad+\left\langle\partial I_{\nabla}(\mathbf{p}), \mathbf{q}\right\rangle=C \int_{\Omega} \nabla \varphi_{1} \cdot \mathbf{q} \mathrm{d} \mathbf{x}, \quad \forall \mathbf{q} \in \mathbf{Q} \\
\mathbf{p}(0)=\mathbf{p}_{0} .
\end{array}\right.
$$

Our goal is thus to integrate in time (4.5) from $t=0$ to $t=+\infty$, in order to capture its steady-state solutions. Those steady-state solutions are also the solutions of (4.2).

The formulation for the regularized problem corresponding to the second Eikonal equation (2.2) is similar: First the problem is regularized and penalized:

$$
\min _{v \in V_{g}^{+}}\left[\frac{\varepsilon_{1}}{2} \int_{\Omega}\left|\nabla^{2} v\right|^{2} \mathrm{~d} \mathbf{x}+J(v)+\frac{1}{4 \varepsilon_{2}} \sum_{i=1}^{2} \int_{\Omega}\left(\left|\frac{\partial v}{\partial x_{i}}\right|^{2}-1\right)^{2} \mathrm{~d} \mathbf{x}\right],
$$

where $V_{g}^{+}=\left\{v \in H^{2}(\Omega), v \geq 0\right.$ in $\Omega, v=g$ on $\left.\Gamma\right\}$, and we denote by $\bar{u}_{\varepsilon} \in V_{g}^{+}$its solution. Then we transfer the nonlinearity burden to $\overline{\mathbf{p}}_{\varepsilon}:=\nabla \bar{u}_{\varepsilon}$ via the following equivalent mixed formulation of (4.6):

$$
\overline{\mathbf{p}}_{\boldsymbol{\varepsilon}}=\arg \min _{\mathbf{q} \in \mathbf{Q}}\left[\frac{1}{2} \int_{\Omega}|\mathbf{q}|^{2} \mathrm{~d} \mathbf{x}-C \int_{\Omega} \nabla \varphi_{1} \cdot \mathbf{q} \mathrm{d} \mathbf{x}+\frac{1}{4 \varepsilon_{2}} \sum_{i=1}^{2} \int_{\Omega}\left(\left|q_{i}\right|^{2}-1\right)^{2} \mathrm{~d} \mathbf{x}+I_{+}(\mathbf{q})\right],
$$

where the functional $I_{+}(\cdot)$ is defined by

$$
I_{+}(\mathbf{q})= \begin{cases}\frac{\varepsilon_{1}}{2} \int_{\Omega}|\nabla \cdot \mathbf{q}| \mathrm{d} \mathbf{x}, & \text { if } \mathbf{q} \in \nabla V_{g}^{+}, \\ +\infty, & \text { if } \mathbf{q} \in \mathbf{Q} \backslash \nabla V_{g}^{+} .\end{cases}
$$

Next, the flow problem is defined in a similar fashion as in (4.5). 


\section{Operator-Splitting Solution}

In order to solve the initial-value problem (4.5), we advocate an operator splitting (Lie-) scheme à la Marchuk-Yanenko for its robustness and simplicity (see, e.g., [7, Chapter 6]). Naturally other schemes are available (like the Strang symmetrized one). Let us denote by $\tau>0$ a time-discretization step and set $t^{n}=n \tau, n=0,1,2, \cdots$. Let $\mathbf{p}^{n}$ be an approximation of $\mathbf{p}\left(t^{n}\right)$. In order to solve (4.5), we advocate the following operator-splitting scheme.

Initialize with

$$
\mathbf{p}^{0}=\mathbf{p}_{0}
$$

For $n \geq 0, \mathbf{p}^{n}$ being known, we compute $\mathbf{p}^{n+\frac{1}{2}}$ and $\mathbf{p}^{n+1}$ successively via the solution of the following problem: Find $\mathbf{p}^{n+\frac{1}{2}} \in \mathbf{Q}$ such that

$$
\int_{\Omega} \frac{\mathbf{p}^{n+\frac{1}{2}}-\mathbf{p}^{n}}{\tau} \cdot \mathbf{q} \mathrm{d} \mathbf{x}+\frac{1}{\varepsilon_{2}} \int_{\Omega}\left(\left|\mathbf{p}^{n+\frac{1}{2}}\right|^{2}-1\right) \mathbf{p}^{n+\frac{1}{2}} \cdot \mathbf{q} \mathrm{d} \mathbf{x}=0
$$

for all $\mathbf{q} \in \mathbf{Q}$. Then, find $\mathbf{p}^{n+1} \in \mathbf{Q}$ such that

$$
\int_{\Omega} \frac{\mathbf{p}^{n+1}-\mathbf{p}^{n+\frac{1}{2}}}{\tau} \cdot \mathbf{q} \mathrm{d} \mathbf{x}+\int_{\Omega} \mathbf{p}^{n+1} \cdot \mathbf{q} \mathrm{d} \mathbf{x}+\left\langle\partial I_{\nabla}\left(\mathbf{p}^{n+1}\right), \mathbf{q}\right\rangle=C \int_{\Omega} \nabla \varphi_{1} \cdot \mathbf{q} \mathrm{d} \mathbf{x}
$$

for all $\mathbf{q} \in \mathbf{Q}$. Actually, (5.2) can be solved point-wise, corresponding thus to an infinite family of low-dimensional optimization problems. On the other hand, (5.3) is a classical linear variational problem written in a mixed form. We are going to discuss in the following sections the solutions of these two problems. The initialization of algorithms (5.1)-(5.3) is the topic of the following remark.

Remark 5.1 (Initialization of the IVP) Choosing sensibly $\mathbf{p}^{0}$ in (5.1) is an important issue in order to reduce the number of time steps (iterations). We thus advocate the following approach: Solve $-\nabla^{2} u_{0}=\frac{C}{|C|}$ in $\Omega$, with $u_{0}=g$ on $\Gamma$. Then, we define $\mathbf{p}^{0}$ by

$$
\mathbf{p}^{0}(\mathbf{x})= \begin{cases}\frac{\nabla u_{0}(\mathbf{x})}{\left|\nabla u_{0}(\mathbf{x})\right|}, & \text { if } \nabla u_{0}(\mathbf{x}) \neq \mathbf{0} \\ \mathbf{0}, & \text { otherwise }\end{cases}
$$

Focusing on the second Eikonal problem (2.2), we can suggest an alternative, albeit similar, splitting procedure that reads as follows: For $n \geq 0, \overline{\mathbf{p}}^{n}$ being known, we compute $\overline{\mathbf{p}}^{n+\frac{1}{2}}$ and $\overline{\mathbf{p}}^{n+1}$ with the following approach: Find $\overline{\mathbf{p}}^{n+\frac{1}{2}} \in \mathbf{Q}$ such that

$$
\begin{aligned}
& \int_{\Omega} \frac{\overline{\mathbf{p}}^{n+\frac{1}{2}}-\overline{\mathbf{p}}^{n}}{\tau} \cdot \mathbf{q} \mathrm{d} \mathbf{x}+\int_{\Omega} \overline{\mathbf{p}}^{n+\frac{1}{2}} \cdot \mathbf{q} \mathrm{d} \mathbf{x} \\
& +\frac{1}{\varepsilon_{2}} \sum_{i=1}^{2} \int_{\Omega}\left(\left|\bar{p}_{i}^{n+\frac{1}{2}}\right|^{2}-1\right) \bar{p}_{i}^{n+\frac{1}{2}} q_{i} \mathrm{~d} \mathbf{x}=C \int_{\Omega} \nabla \varphi_{1} \cdot \mathbf{q} \mathrm{d} \mathbf{x}
\end{aligned}
$$

for all $\mathbf{q} \in \mathbf{Q}$. Then, find $\overline{\mathbf{p}}^{n+1} \in \mathbf{Q}$ such that

$$
\int_{\Omega} \frac{\overline{\mathbf{p}}^{n+1}-\overline{\mathbf{p}}^{n+\frac{1}{2}}}{\tau} \cdot \mathbf{q} \mathrm{d} \mathbf{x}+\left\langle\partial I_{+}\left(\overline{\mathbf{p}}^{n+1}\right), \mathbf{q}\right\rangle=0
$$

for all $\mathbf{q} \in \mathbf{Q}$. 


\subsection{Solution of the local optimization problems}

Problem (5.2) does not involve any derivatives. Thus it can be solved locally for almost every point $\mathbf{x} \in \Omega$. Rewriting (5.2) locally, we see that $\mathbf{p}^{n+\frac{1}{2}}(\mathbf{x})$ verifies

$$
\mathbf{p}^{n+\frac{1}{2}}(\mathbf{x})\left[\left(1-\frac{\tau}{\varepsilon_{2}}\right)+\frac{\tau}{\varepsilon_{2}}\left|\mathbf{p}^{n+\frac{1}{2}}(\mathbf{x})\right|^{2}\right]=\mathbf{p}^{n}(\mathbf{x}), \quad \text { a.e. } \mathbf{x} \text { on } \Omega \text {. }
$$

Taking the canonical Euclidean norm of both sides of the vector-valued equation (5.6), it follows that $\left|\mathbf{p}^{n+\frac{1}{2}}(\mathbf{x})\right|$ is the solution of the following cubic equation:

$$
\frac{\tau}{\varepsilon_{2}} z^{3}+\left(1-\frac{\tau}{\varepsilon_{2}}\right) z=\left|\mathbf{p}^{n}(\mathbf{x})\right| .
$$

This problem has a unique solution, necessarily non-negative, if

$$
\tau \leq \varepsilon_{2}
$$

Once $\left|\mathbf{p}^{n+\frac{1}{2}}(\mathbf{x})\right|$ is known, we obtain $\mathbf{p}^{n+\frac{1}{2}}(\mathbf{x})$ from (5.6), by setting

$$
\mathbf{p}^{n+\frac{1}{2}}(\mathbf{x})=\left(\frac{1}{\left(1-\frac{\tau}{\varepsilon}\right)+\frac{\tau}{\varepsilon}\left|\mathbf{p}^{n+\frac{1}{2}}(\mathbf{x})\right|^{2}}\right) \mathbf{p}^{n}(\mathbf{x})
$$

To solve the nonlinear equation (5.7), we advocate the Newton-Raphson method starting from the initial guess $z^{0}=1$.

On the other hand, (5.4) is even easier to solve than (5.2) since it leads to a sequence of pairs of pointwise scalar cubic equations reading as follows:

$$
\left(1+\tau-\frac{\tau}{\varepsilon_{2}}\right) p_{i}^{n+\frac{1}{2}}+\frac{\tau}{\varepsilon_{2}}\left(\bar{p}_{i}^{n+\frac{1}{2}}\right)^{3}=\bar{p}_{i}^{n}+\tau C \frac{\partial \varphi_{1}}{\partial x_{i}}, \quad i=1,2 .
$$

All this cubic equations are of the form

$$
\left(1+\tau-\frac{\tau}{\varepsilon_{2}}\right) z+\frac{\tau}{\varepsilon_{2}} z^{3}=b
$$

which has a unique solution in $\mathbb{R}$ if $\tau \leq \varepsilon_{2}$. To solve equation (5.8), we advocate, again, the Newton-Raphson method (initialized by $b$, for example).

In practice, after an appropriate finite-difference or finite element approximation of (5.2), we have to solve at each time step a cubic equation like (5.7) for each grid point or triangle of the associated finite-difference or finite element grid. Similar to (5.4), but this time the number of cubic equations to solve at each time step is doubled. Since $\Omega$ is bounded in $\mathbb{R}^{2}$, the number of such cubic equations is of the order of $h^{-2}$, where $h$ is a space discretization step.

\subsection{Solution of the linear variational problems}

We can easily prove that the solution $\mathbf{p}^{n+1}$ of (5.3) corresponds, after setting $\nabla u^{n+1}:=$ $\mathbf{p}^{n+1}$, to the solution of the well-posed linear biharmonic problem: Find $u^{n+1} \in H^{2}(\Omega) \cap H_{g}^{1}(\Omega)$ satisfying

$$
\begin{aligned}
& \int_{\Omega} \frac{\nabla u^{n+1}-\mathbf{p}^{n+\frac{1}{2}}}{\tau} \cdot \nabla v \mathrm{~d} \mathbf{x}+\varepsilon_{1} \int_{\Omega}\left(\nabla^{2} u^{n+1}\right)\left(\nabla^{2} v\right) \mathrm{d} \mathbf{x} \\
& +\int_{\Omega} \nabla u^{n+1} \cdot \nabla v \mathrm{~d} \mathbf{x}=C \int_{\Omega} v \mathrm{~d} \mathbf{x}, \quad \forall v \in H^{2}(\Omega) \cap H_{0}^{1}(\Omega) .
\end{aligned}
$$


(5.9) is nothing but a variational formulation of the following bi-harmonic problem:

$$
\left\{\begin{array}{l}
-(1+\tau) \nabla^{2} u^{n+1}+\varepsilon_{1} \tau \nabla^{4} u^{n+1}=\tau C-\nabla \cdot \mathbf{p}^{n+\frac{1}{2}} \quad \text { in } \Omega, \\
u^{n+1}=g \quad \text { on } \Gamma, \quad \nabla^{2} u^{n+1}=0 \quad \text { on } \Gamma .
\end{array}\right.
$$

Such a biharmonic problem can be factored as the following system of two well-posed secondorder linear elliptic problems:

$$
\begin{aligned}
& \left\{\begin{array}{l}
(1+\tau) w^{n+1}-\varepsilon_{1} \tau \nabla^{2} w^{n+1}=\tau C-\nabla \cdot \mathbf{p}^{n+\frac{1}{2}} \text { in } \Omega, \\
w^{n+1}=0 \text { on } \Gamma,
\end{array}\right. \\
& \left\{\begin{array}{l}
-\nabla^{2} u^{n+1}=w^{n+1} \\
u^{n+1}=g \text { on } \Gamma .
\end{array}\right.
\end{aligned}
$$

Many methods are available for the numerical solution of the two above elliptic boundary value problems (see, e.g., [7] and the references therein).

Concerning the solution of (5.5), the non-negativity constraint in $V_{g}^{+}$implies that the analogue to (5.9) is actually a variational inequality of the obstacle type [8] that reads as the following problem: Find $\bar{u}^{n+1} \in V_{g}^{+}$satisfying

$$
\begin{aligned}
& \int_{\Omega} \nabla \bar{u}^{n+1} \cdot \nabla\left(v-\bar{u}^{n+1}\right) \mathrm{d} \mathbf{x}+\tau \varepsilon_{1} \int_{\Omega}\left(\nabla^{2} \bar{u}^{n+1}\right) \nabla^{2}\left(v-\bar{u}^{n+1}\right) \mathrm{d} \mathbf{x} \\
\geq & \int_{\Omega} \overline{\mathbf{p}}^{n+\frac{1}{2}} \cdot \nabla\left(v-\bar{u}^{n+1}\right) \mathrm{d} \mathbf{x}, \quad \forall v \in V_{0}^{+} .
\end{aligned}
$$

The finite element solution of the elliptic system (5.11)-(5.12) for two-dimensional domains $\Omega$ of arbitrary shape is routine (see, e.g., [7] and the references therein), which is the most complicated part of our methodology. On the other hand, solving (5.13) is a more complicated issue which is addressed in $[2,7]$. Considerations of boundary layer thickness suggest to take $\tau \varepsilon_{1}$ of the order of $h^{2}$, roughly, for both types of problems.

\section{Numerical Experiments}

In this section, numerical experiments are presented to validate our methodology for both types of Eikonal equations. We consider here only the unit square $\Omega=(0,1)^{2}$.

\subsection{Homogeneous boundary conditions (recovery of the distance function)}

The first numerical example corresponds to the homogeneous case $g=0$ in (2.1). The maximal solution of (2.1) is the distance function $\mathbf{x} \rightarrow \delta(\mathbf{x}, \Gamma)$ (distance of $\mathbf{x}$ to the boundary $\Gamma$ of $\Omega$ ); it is given here by

$$
u_{\max }\left(x_{1}, x_{2}\right)=\min \left\{x_{1}, 1-x_{1}, x_{2}, 1-x_{2}\right\}, \quad \forall \mathbf{x}=\left(x_{1}, x_{2}\right) \in \Omega .
$$

Since $g=0$, the minimal solution of (2.1) is just the opposite of the maximal one, i.e., $u_{\min }=-u_{\max }$. For our computations, we have used $C=10$. The finite element mesh we used is a structured triangulation $\mathcal{T}_{h}$ of the "British flag" type. For the solution of the local nonlinear problems, we have used the Newton's method with the stopping criterion tolerance equal to $10^{-4}$; with this tolerance, the Newton's algorithm was always converging in less than 10 iterations, typically. 
Remark 6.1 In order to speed up the convergence and improve accuracy, we update $\varepsilon_{2}$ and $\tau$ at each time step of the operator-splitting scheme as follows: $\tau^{n+1}=\xi \tau^{n}$ and $\varepsilon_{2}^{n+1}=\xi \varepsilon_{2}^{n}$, with $\xi \in(0,1)$. Here $\tau^{n}$ (resp. $\varepsilon_{2}^{n}$ ) thus denotes the value of $\tau$ (resp. $\varepsilon_{2}$ ) at the $n$th time step of the operator-splitting scheme. For our computations, we took $\varepsilon_{1}=10^{-3}, \varepsilon_{2}^{0}=0.1$, $\tau^{0}=8 \times 10^{-2}$ and $\xi=0.9$.

In Figure 1, we have reported the graphs of the computed maximal and minimal solutions, their contours, and the "contours" of $\left|\nabla u_{\max , h}\right|$ and $\left|\nabla u_{\min , h}\right|$ obtained with $h=\frac{1}{20}$ and 100 time steps of the operator-splitting scheme (5.2)-(5.3). The latter shows that the Eikonal equation is satisfied, up to rounding errors and mesh effects.
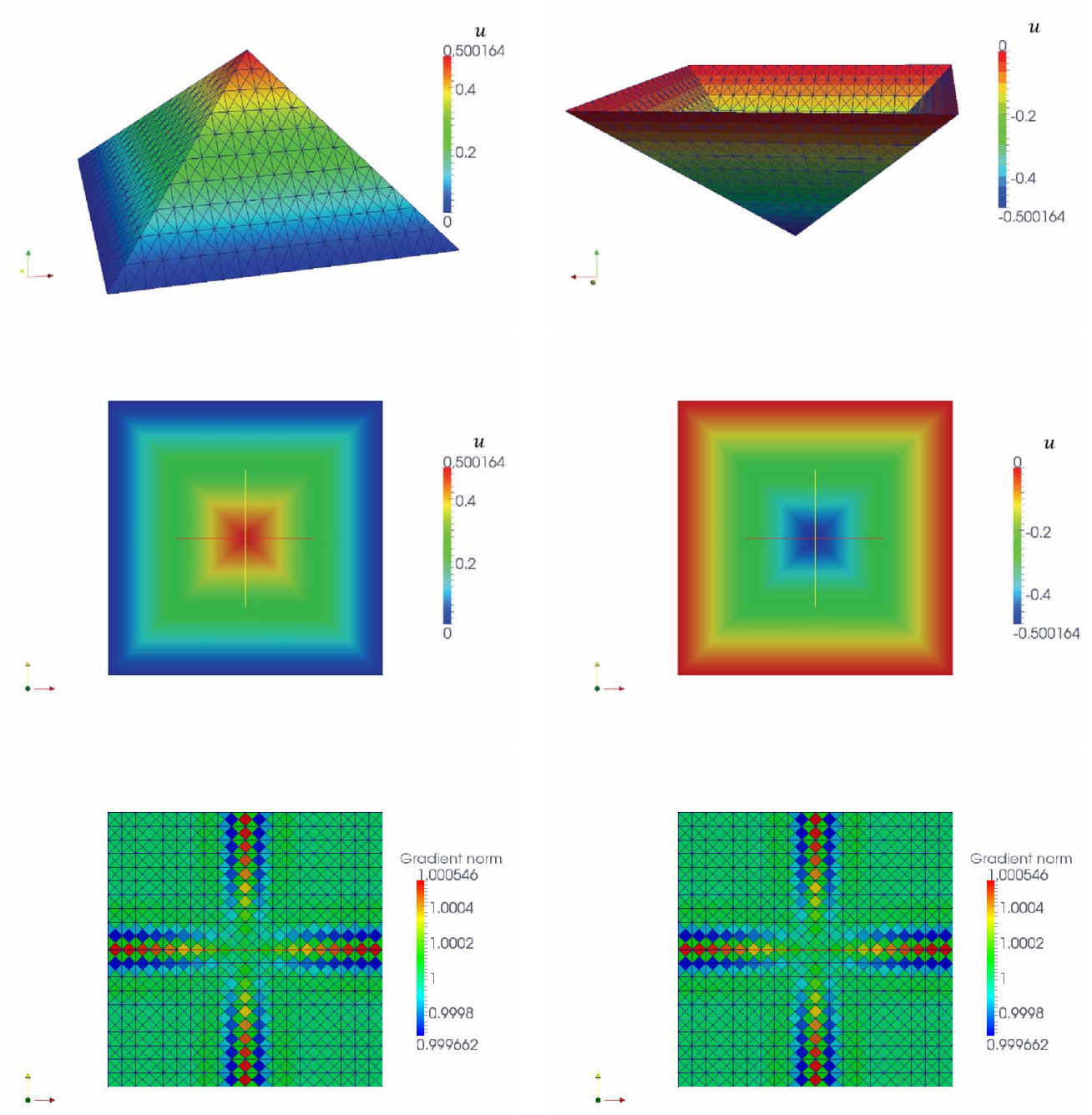

Figure 1 Distance function on the unit square $(g=0)$. Approximation $u_{h}$ of the solution of the Eikonal equation ( $h=\frac{1}{20}$, after 100 iterations). Left: Maximal solution; right: Minimal solution. First row: Graph of $u_{h}$; second row: Contour of $\left|u_{h}\right|$; third row: Piecewise constant approximation $\left|\nabla u_{h}\right|$. 
Convergence results are visualized in Figure 2, which show the $\mathcal{O}\left(h^{\frac{1}{2}}\right)$ approximation error for both the $L^{2}$ and $H^{1}$-norms, whenever $u=u_{\max }$ or $u=u_{\min }$. The low regularity of the solution (belonging to $W^{1, \infty}(\Omega) \cap H^{s}(\Omega)$ for all $s<\frac{3}{2}$ ) explains why we do not obtain the usual $\mathcal{O}\left(h^{2}\right)$ and $\mathcal{O}(h)$ approximation errors.
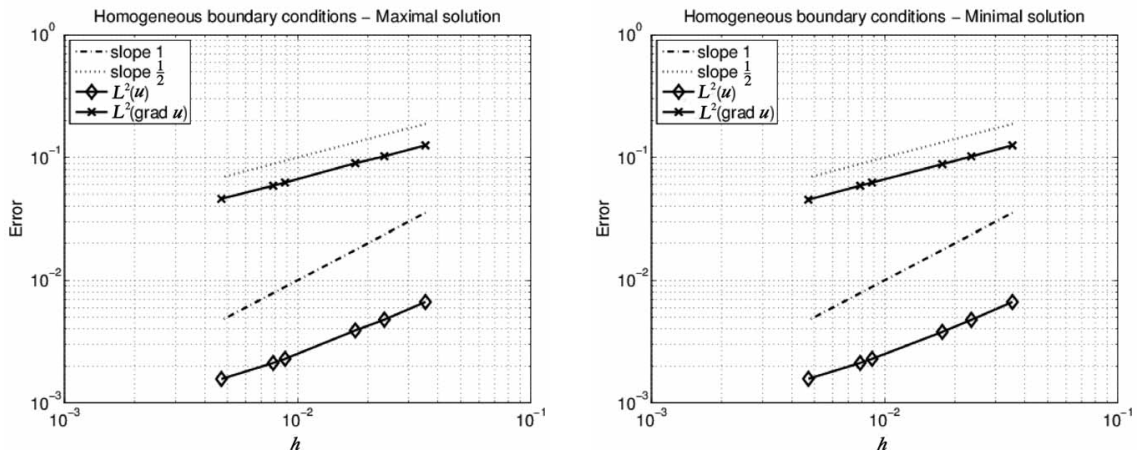

Figure 2 Distance function on the unit square $(g=0$; linear biharmonic regularization). Approximation errors $\left\|u_{h}-u_{\min }\right\|_{0 h}$ and $\left\|\nabla\left(u_{h}-u_{\min }\right)\right\|$ (resp. $\left\|u_{h}-u_{\max }\right\|_{0 h}$ and $\left.\left\|\nabla\left(u_{h}-u_{\max }\right)\right\|\right)$ versus $h$ (100 outer iterations). Left: Maximal solution; right: Minimal solution.

If we look for the solution of (2.2) with $g=0$, the maximal solutions do not exist. Figure 3, visualizes the graphs and contours of the discrete solution with $h=\frac{1}{512}$ and $h=\frac{1}{1024}$. These figures show that new structures appear when refining the mesh, showing a fractal behavior.
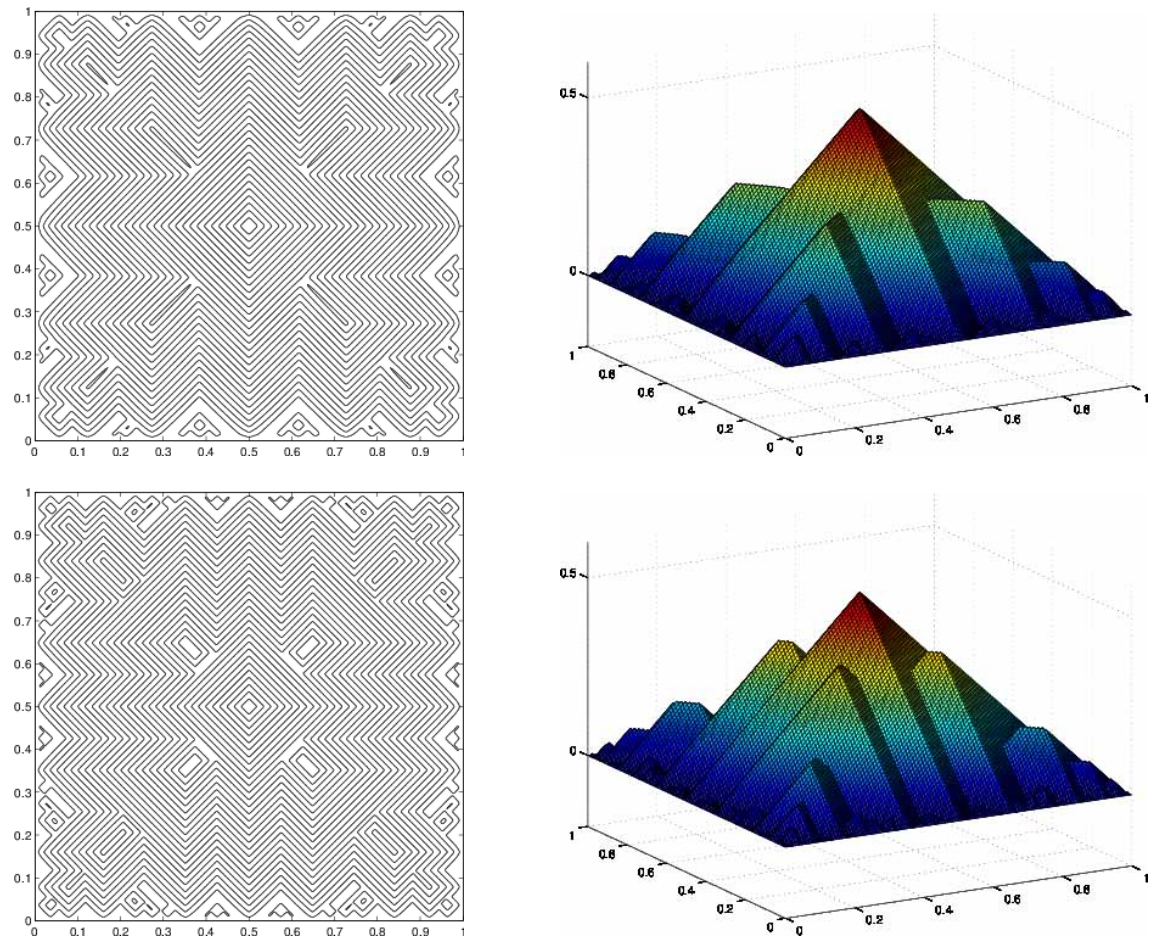

Figure 3 Contours and graphs of the discrete maximal solution $u_{h}$ of the Eikonal problem (3.8) $\left(h=\frac{1}{512}\right.$ and $\left.h=\frac{1}{1024}\right)$. 


\subsection{Non-homogeneous boundary conditions}

The numerical example we consider now concerns the search of the maximal and minimal solutions of the Eikonal equation (2.1) when

$$
\Omega=(0,1)^{2}
$$

and $g$ is defined by

$$
g\left(x_{1}, x_{2}\right)= \begin{cases}0 & \text { on } \Gamma_{1} \cup \Gamma_{3} \cup \Gamma_{4}, \\ \min \left(x_{2}, 1-x_{2}\right) & \text { on } \Gamma_{2},\end{cases}
$$

where

$$
\begin{aligned}
& \Gamma_{1}=[0,1] \times\{0\}, \\
& \Gamma_{2}=\{1\} \times(0,1), \\
& \Gamma_{3}=[0,1] \times\{1\}, \\
& \Gamma_{4}=\{0\} \times(0,1) .
\end{aligned}
$$

The corresponding maximal solution is given by

$$
u_{\max }\left(x_{1}, x_{2}\right)=\min \left(x_{1}, x_{2}, 1-x_{2}\right) .
$$

On the other hand, the closed form of the minimal solution is given (with $\mathbf{x}=\left\{x_{1}, x_{2}\right\}$ ) by

$$
u_{\min }\left(x_{1}, x_{2}\right)=\max \left\{-x_{1},-x_{2}, x_{2}-1, \frac{1}{2}-\sqrt{\left(x_{1}-1\right)^{2}+\left(x_{2}-\frac{1}{2}\right)^{2}}\right\} .
$$

In order to solve numerically this new test problem, we take

(i) $\varepsilon_{1}=0.001, \varepsilon_{2}^{0}=0.25, \tau^{0}=0.2, C=10$ and $\xi=0.9$ if $h=\frac{1}{40}$;

(ii) $\varepsilon_{1}=0.001, \varepsilon_{2}=0.25, \tau=0.2, C=100$ and $\xi=0.9$ if $h=\frac{1}{120}$.

To obtain the minimal solution, we set

$$
C=-100
$$

in both cases. Figure 4 visualizes the discrete maximal and minimal solutions.

Looking at $\left|\nabla u_{h}\right|$, one can observe that it is almost everywhere equal to one. The exception is in the neighborhood of an edge, due to the mesh effects. Note that, for the maximal solution for instance, the approximation error due to the mesh is only present when mesh edges are perpendicular to the solution's edges.

Figure 5 visualizes the convergence properties of the computed approximate solutions obtained with various types of discretizations of the unit square. All types of meshes produce essentially the same results, suggesting $\mathcal{O}\left(h^{\frac{1}{2}}\right)$ for $\left\|u_{h}-u\right\|_{0 h}$. When the edges of the mesh follow the lines of discontinuity of the gradient of the solution, the convergence order is actually closer to $\mathcal{O}(h)$, suggesting some kind of super-convergence. 

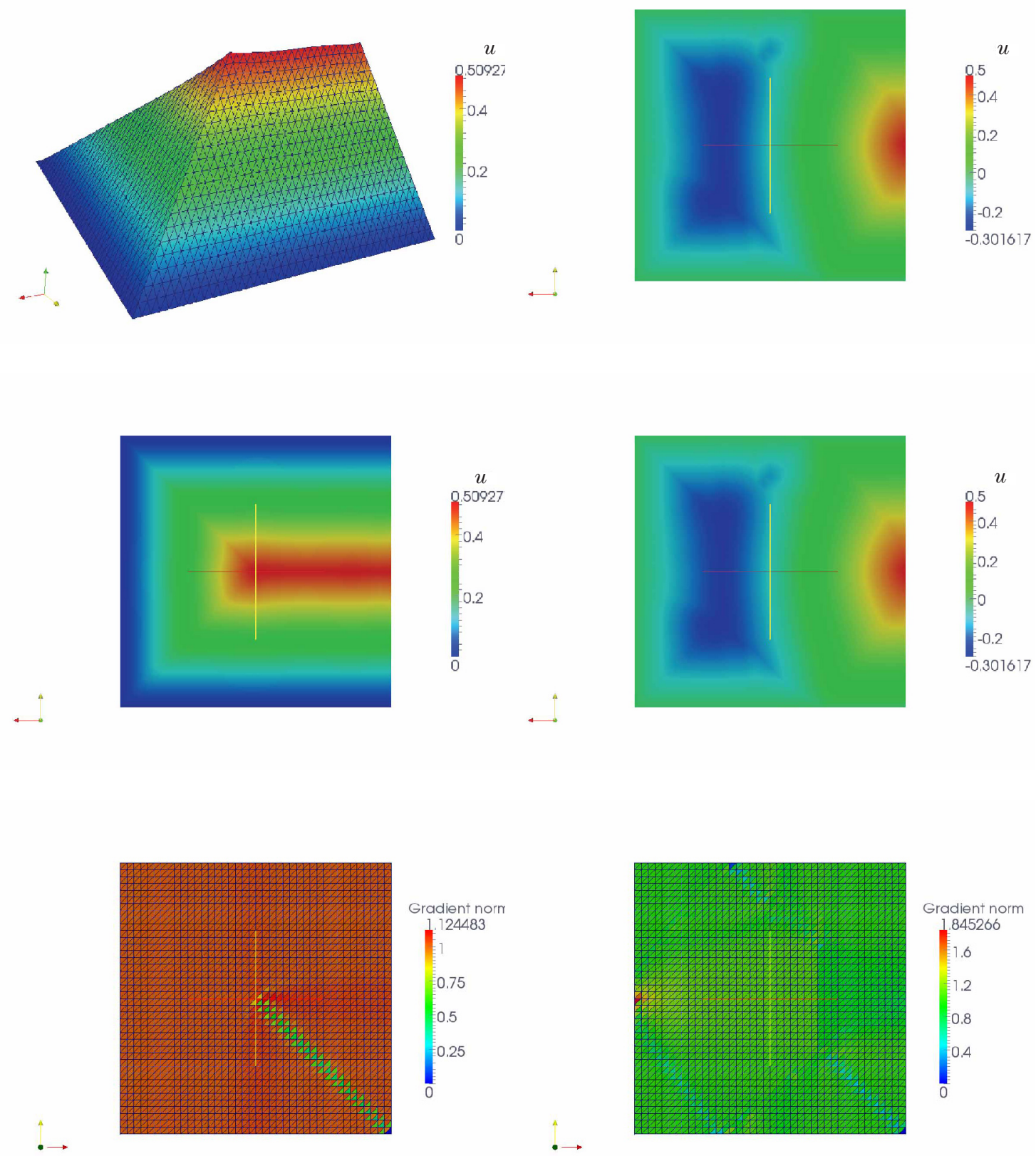

Figure 4 Non-homogeneous boundary conditions. Approximation $u_{h}$ of the solution of the Eikonal equation ( $h=\frac{1}{20}$, after 100 iterations). First row: Graph of $u_{h}$; second row: Contour of $\left|u_{h}\right|$; third row: Visualization of $\left|\nabla u_{h}\right|$. Left: Maximal solution; right: Minimal solution. 

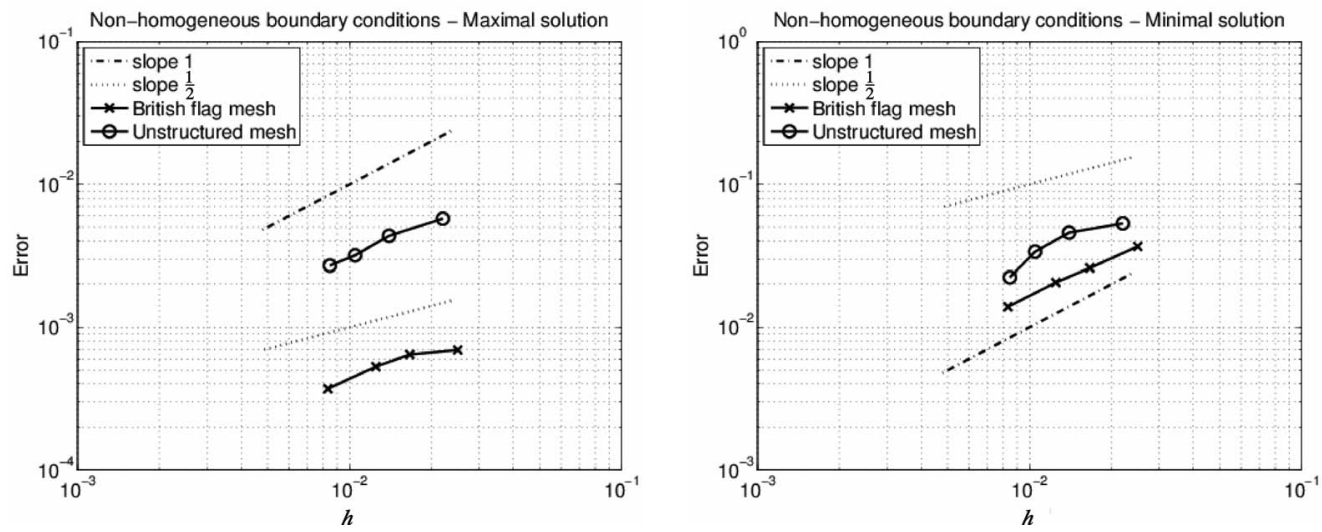

Figure 5 Non-homogeneous boundary conditions. Approximation errors $\left\|u_{h}-u_{\min }\right\|_{0 h}$ (resp. $\left\|u_{h}-u_{\max }\right\|_{0 h}$ ) versus $h$ for various types of discretizations (British flag mesh, and unstructured mesh). Left: Maximal solution; right: Minimal solution.

\section{Conclusions}

A numerical approach based on elliptic solvers has been presented to compute numerical approximations of two scalar steady Eikonal equations. A variational approach allows to calculate the maximal/minimal solutions. Numerical algorithms rely on appropriate penalization and regularization techniques, coupled with an operator-splitting algorithm for the corresponding initial value problem. Perspectives include the use of such an elliptic solver approach to vectorial Eikonal problems arising in origami modeling.

Acknowledgement The authors would like to thank Prof. B. Dacorogna (EPFL) for fruitful discussions and suggesting the investigation of such non-smooth problems.

\section{References}

[1] Caffarelli, L. and Crandall, M. G., Distance functions and almost global solutions of Eikonal equations, Comm. Partial Differential Equations, 3, 2010, 391-414.

[2] Dacorogna, B., Glowinski, R., Kuznetzov, Y. and Pan, T.-W., On a Conjuguate Gradient/Newton/Penalty Method for the Solution of Obstacle Problems, Application to the Solution of an Eikonal System with Dirichlet Boundary Conditions, Conjugate Gradient Algorithms and Finite Element Methods, M. Kř́žek, P. Neittaanmäki, R. Glowinski and S. Korotov (eds.), Springer-Verlag, Berlin, Heidelberg, 2004, 263-283.

[3] Dacorogna, B., Glowinski, R. and Pan, T.-W., Numerical methods for the solution of a system of Eikonal equations with Dirichlet boundary conditions, C. R. Acad. Sci. Paris, Sér. I, 336, 2003, 511-518.

[4] Dacorogna, B. and Marcellini, P., Implicit Partial Differential Equations, Birkhaüser, Basel, 1999.

[5] Dacorogna, B. and Marcellini, P. and Paolini, E., Lipschitz-continuous local isometric immersions: Rigid maps and origami, Journal Math. Pures Appl., 90, 2008, 66-81.

[6] Dacorogna, B., Marcellini, P. and Paolini, E., Origami and partial differential equations, Notices of the American Math. Soc., 57, 2010, 598-606.

[7] R. Glowinski, Finite element method for incompressible viscous flow, Volume IX of Handbook of Numerical Analysis, P. G. Ciarlet, J. L. Lions (eds.), Elsevier, Amsterdam, 2003, 3-1176.

[8] Glowinski, R., Kuznetzov, Y. and Pan, T.-W., A penalty/Newton/conjugate gradient method for the solution of obstacle problems, C. R. Acad. Sci. Paris, Sér. I, 336, 2003, 435-440.

[9] Kimmel, R. and Sethian, J. A., Computing geodesic paths on manifolds, Proceedings of National Academy of Sciences, 95(15), 1998, 8431-8435. 
[10] Malladi, R. and Sethian, J., A unified approach to noise removal, image enhancement, and shape recovery, IEEE Trans. on Image Processing, 5(11), 1996, 1554-1568.

[11] Qin, F., Luo, Y., Olsen, K., et al., Finite-difference solution of the Eikonal equation along expanding wavefronts, Geophysics, 57(3), 1992, 478-487.

[12] Rockafellar, R. T., Convex Analysis, Princeton University Press, Princeton, 1996.

[13] Sethian, J., Level Set Methods and Fast Marching Methods, Cambridge University Press, Cambridge, 2002 .

[14] Sethian, J. A., Fast marching methods, SIAM Rev., 41(2), 1999, 199-235. 